\title{
Minimally Invasive Techniques for an Intersphincteric Resection and Lateral Pelvic Lymph Node Dissection in Rectal Cancer
}

\author{
Jung Wook Huh \\ Department of Surgery, Samsung Medical Center, Sungkyunkwan University School of Medicine, Seoul, Korea
}

A proper total mesorectal excision (TME) technique is paramount to the successful treatment of locally advanced rectal cancer irrespective of operative method, including open and minimally-invasive approaches. The use of minimally-invasive surgery is of growing interest for the treatment of rectal cancer. The results of a recent, multicenter, randomized COREAN trial and of the COlorectal cancer Laparoscopic or Open Resection (COLOR II) trial suggested that high-quality laparoscopic TME in rectal cancer could be safely achieved through proper patient selection when performed by a skilled surgeon $[1,2]$. Moreover, robot-assisted rectal surgery has recently been introduced as a minimallyinvasive alternative. Several retrospective analyses have suggested that robotic surgery has several technical advantages and may lead to favorable patient outcomes, although it still has a few drawbacks, including a long operation time and high cost [3-5].

The potential benefits of laparoscopic colorectal surgery compared with open surgery include faster recovery, lower morbidity, less pain, and a shorter hospital stay, and these can be achieved without compromising oncologic outcomes [6]. Although a laparoscopic colorectal resection is now an established treatment for colorectal cancer, a belief exists that laparoscopic colorectal surgery is not suitable for tumors that have invaded adjacent organs or structures due to its having high morbidities, conversion rates, and questionable oncologic outcomes. Especially, an open resection for an intersphincteric resection (ISR) and a lateral pelvic lymph node dissection (LPND) in rectal cancer remains a very troublesome and demanding procedure; therefore, it would become even more difficult when performed laparoscopically. More

Correspondence to: Jung Wook Huh, M.D.

Department of Surgery, Samsung Medical Center, Sungkyunkwan University School of Medicine, 81 Irwon-ro, Gangnam-gu, Seoul 135-710, Korea

Tel: +82-2-3410-1826, Fax: +82-2-3410-6980

E-mail: jungwook.huh@gmail.com

(c) 2014 The Korean Society of Coloproctology

This is an open-access article distributed under the terms of the Creative Commons Attribution NonCommercial License (http://creativecommons.org/licenses/by-nc/3.0) which permits unrestricted noncommercial use, distribution, and reproduction in any medium, provided the original work is properly cited. recently, with advances in modern laparoscopic technology, a minimally-invasive laparoscopic resection for these circumstances has been reported. I previously suggested that a laparoscopic resection for colorectal cancer could be achieved with low rates of conversion and morbidity even in patients with a preoperativelysuspected T4 tumor, which is associated with the acceptable perioperative outcomes and the disease-free survival rates when compared with an open resection [7]. Moreover, I believe that patients with demanding conditions, such as duodenal or pancreatic invasion in right-sided colon cancer, complex pelvic irregularities or bladder invasion in rectal cancer, or severe intra-abdominal adhesion due to multiple previous surgeries, should immediately be considered for open surgery or looked at laparoscopically with a high suspicion that the surgery would be converted to an open procedure [7]. Although proper patient selection is one of the most important factors for minimally-invasive surgery, many skilled surgeons who have overcome the learning curve for minimally-invasive surgery tend to expand the operative indications for a minimally-invasive procedure. Therefore, I would like to review the more complex minimally-invasive procedures for patients with advanced rectal cancer.

A number of recent retrospective studies have indicated that more complex minimally-invasive procedures, such as ISR and LPND are frequently performed by skilled surgeons [5, 8-11]. Park et al. [10] suggested that a laparoscopic ISR group had a shorter hospital stay and shorter time to bowel movement compared with a matched open ISR group; the local recurrence rate and the 3-year disease-free survival were similar between the two groups during a median follow-up period of 34 months. Lim et al. [11] revealed that 101 patients who underwent a laparoscopic ISR after preoperative chemoradiotherapy for rectal cancer demonstrated a relatively preserved Wexner continence score of 7.5 after ileostomy closure, that observation being based on a questionnaire on postoperative anal function. Moreover, some skilled surgeons believe that a robotic ISR is also feasible and safe for patients with low-lying rectal cancer. In a retrospective comparative study of 84 coloanal anastomoses, Baek et al. [5] showed a lower 
incidence of conversion to open surgery (2.1\% vs. $16.2 \%)$ and a shorter hospital stay (9 days vs. 11 days) for the robotic than for the laparoscopic coloanal anastomosis group.

In an era of frequent advances in preoperative chemoradiotherapy for locally-advanced rectal cancer, the role of an extended LPND following a TME for this disease is still controversial. A recent meta-analysis comparing an extended and a nonextended LPND in patients with rectal cancer revealed no overall difference in cancer-specific outcomes [8]. For patients with persistent enlargement of lymph nodes even after preoperative chemoradiotherapy, many surgeons selectively performed a LPND. Park et al. [9] also indicated that the surgical strategy regarding a LPND, in addition to a TME, has changed due to the introduction of preoperative chemoradiotherapy. The authors suggested several potential benefits of a laparoscopic LPND over the open technique. First, laparoscopic surgery provided magnified and clear vision of the smallest structure in the narrow pelvis, and protecting the autonomic nerve plexus was easier. Second, the use of an advanced energy device during surgery resulted in favorable early postoperative outcomes. Finally, laparoscopic surgeries involve only a small abdominal incision and potentially reduce the risk of peritoneal adhesion. Robotic surgery can potentially provide articulated movement, elimination of tremors, and a magnified threedimensional view in a limited space. In a series of 8 consecutive robotic LPNDs, Park et al. [12] reported that the mean operating time for an extended LPND was 38 minutes (range, 20 to $51 \mathrm{~min}$ utes) and that no LPND-related morbidities had occurred.

In conclusion, the potential benefits of minimally-invasive approaches in patients who undergo an ISR, as well as an extended LPND, can be achieved in a setting of high patient selectivity with surgeons experienced in minimally-invasive surgical techniques. Well-organized, prospective, randomized studies are needed to establish the advantage of minimally-invasive approaches over open surgery in the treatment of patients with locally-advanced rectal cancer.

\section{REFERENCES}

1. Kang SB, Park JW, Jeong SY, Nam BH, Choi HS, Kim DW, et al. Open versus laparoscopic surgery for mid or low rectal cancer after neoadjuvant chemoradiotherapy (COREAN trial): short-term outcomes of an open-label randomised controlled trial. Lancet
Oncol 2010;11:637-45.

2. van der Pas MH, Haglind E, Cuesta MA, Furst A, Lacy AM, Hop WC, et al. Laparoscopic versus open surgery for rectal cancer (COLOR II): short-term outcomes of a randomised, phase 3 trial. Lancet Oncol 2013;14:210-8.

3. Kang J, Yoon KJ, Min BS, Hur H, Baik SH, Kim NK, et al. The impact of robotic surgery for mid and low rectal cancer: a casematched analysis of a 3-arm comparison: open, laparoscopic, and robotic surgery. Ann Surg 2013;257:95-101.

4. Kim JC, Yang SS, Jang TY, Kwak JY, Yun MJ, Lim SB. Open versus robot-assisted sphincter-saving operations in rectal cancer patients: techniques and comparison of outcomes between groups of 100 matched patients. Int J Med Robot 2012;8:468-75.

5. Baek SJ, Al-Asari S, Jeong DH, Hur H, Min BS, Baik SH, et al. Robotic versus laparoscopic coloanal anastomosis with or without intersphincteric resection for rectal cancer. Surg Endosc 2013; 27:4157-63.

6. Guillou PJ, Quirke P, Thorpe H, Walker J, Jayne DG, Smith AM, et al. Short-term endpoints of conventional versus laparoscopic-assisted surgery in patients with colorectal cancer (MRC CLASICC trial): multicentre, randomised controlled trial. Lancet 2005; 365:1718-26.

7. Huh JW, Kim HR. The feasibility of laparoscopic resection compared to open surgery in clinically suspected T4 colorectal cancer. J Laparoendosc Adv Surg Tech A 2012;22:463-7.

8. Georgiou P, Tan E, Gouvas N, Antoniou A, Brown G, Nicholls RJ, et al. Extended lymphadenectomy versus conventional surgery for rectal cancer: a meta-analysis. Lancet Oncol 2009;10:1053-62.

9. Park JS, Choi GS, Lim KH, Jang YS, Kim HJ, Park SY, et al. Laparoscopic extended lateral pelvic node dissection following total mesorectal excision for advanced rectal cancer: initial clinical experience. Surg Endosc 2011;25:3322-9.

10. Park JS, Choi GS, Jun SH, Hasegawa S, Sakai Y. Laparoscopic versus open intersphincteric resection and coloanal anastomosis for low rectal cancer: intermediate-term oncologic outcomes. Ann Surg 2011;254:941-6.

11. Lim SW, Huh JW, Kim YJ, Kim HR. Laparoscopic intersphincteric resection for low rectal cancer. World J Surg 2011;35:2811-7.

12. Park JA, Choi GS, Park JS, Park SY. Initial clinical experience with robotic lateral pelvic lymph node dissection for advanced rectal cancer. J Korean Soc Coloproctol 2012;28:265-70. 